Artículo científico

Volumen 32(1):19-33. Enero-abril, 2021

e-ISSN 2215-3608, doi:10.15517/am.v32i1.40130

https://www.revistas.ucr.ac.cr/index.php/agromeso

\title{
Métodos de reproducción y parámetros reproductivos de cebuínos con registro genealógico en Costa Rica ${ }^{1}$
}

\section{Breeding methods and reproductive parameters of Zebu cattle with genealogical records in Costa Rica}

\author{
Marilyn Vásquez-Loaiza², Roger Molina-Coto
}

1 Recepción: 19 de enero, 2020. Aceptación: 10 de junio, 2020. Este trabajo formó parte del proyecto de graduación de la primera autora para la licenciatura en Ingeniería Agronómica con énfasis en Zootecnia de la Universidad de Costa Rica.

2 Asociación de Criadores de Ganado Cebú de Costa Rica. San José, Costa Rica. marilyntatiana.vasquez@gmail.com (https://orcid.org/00000002-7267-603X).

3 Universidad de Costa Rica, Facultad de Ciencias Agroalimentarias. Escuela de Zootecnia, San José, Costa Rica. roger.molina@ucr.ac.cr (autor para la correspondencia, https://orcid.org/0000-0003-3844-2587).

\section{Resumen}

Introducción. En Costa Rica, la población de ganado con registro genealógico de la Asociación de Criadores de Ganado Cebú (ASOCEBÚ), no contaba con una caracterización reproductiva ni con parámetros como edad al primer parto (EPP) o intervalo entre partos (IEP), lo cual limita el análisis, progreso genético y productivo de las razas. Objetivo. Caracterizar los métodos de reproducción utilizados en las razas puras cebuínas y calcular la EPP e IEP de una población Brahman gris pura en Costa Rica. Materiales y métodos. Se utilizó la base de datos de la ASOCEBÚ Costa Rica, actualizada a marzo 2018. Se analizaron 10995 registros de las razas Brahman Gris, Nelore, Brahman Rojo, Gyr, Guzerat e Indubrasil. Se calculó la distribución de los animales según los métodos de reproducción utilizados. Del 32,7 \% de la población Brahman gris en ASOCEBÚ, proveniente de las regiones Huetar Atlántica, Brunca y Chorotega, se calculó la EPP e IEP. Resultados. La monta natural, fue el método de reproducción más utilizado $(57,8 \%)$ seguido por la inseminación artificial $(26 \%)$ y transferencia de embriones $(16,2 \%)$. El promedio de EPP en la raza Brahman Gris fue de 41,4 meses con un IEP de 548,9 días. Conclusiones. Es necesario seleccionar animales con mayor precocidad sexual y darles las condiciones para disminuir la EPP e IEP. El uso de la inseminación artificial, mejoró el desempeño reproductivo de los animales Brahman puros. Las asociaciones de bovinos de raza pueden promover la mejora de los animales que registran a través de controles y análisis de parámetros zootécnicos.

Palabras clave: cebú, edad a primer parto, intervalo entre partos, ganado puro.

\begin{abstract}
Introduction. In Costa Rica, the population of cattle with a genealogical record of the Association of Breeders of Zebu Cattle (ASOCEBU), did not have a reproductive characterization or parameters such as age at first calving (EPP) or calving interval (IEP), which limits the analysis, genetic, and productive progress of the breeds. Objective. Characterize the types of methods used to breed pure zebu breeds and calculate the EPP and IEP of a pure gray Brahman population in Costa Rica. Materials and methods. The ASOCEBÚ database from Costa Rica, updated to
\end{abstract}


March, 2018 was used. 10,995 records of the Gray Brahman, Nelore, Red Brahman, Gyr, Guzerat, and Indubrasil breeds were analyzed. The distribution of the animals was calculated according to the types of reproduction used. Of the $32.7 \%$ of the gray Brahman population in ASOCEBÚ from the Huetar Atlántica, Brunca, and Chorotega regions, the EPP and IEP were calculated. Results. The natural mounting was the type of most used method (57.8 \%) for the reproduction of zebu animals followed by artificial insemination (26\%) and embryo transfer (16.2\%). The average EPP in the Gray Brahman breed was 41.4 months with an IEP of 548.9 days. Conclusions. It is necessary to select animals with greater sexual precocity and give them the conditions to reduce PPE and IEP. The use of artificial insemination improved the reproductive performance of pure Brahman animals. Breed associations can promote the improvement of animals they register through records and analysis of zootechnical parameters.

Keywords: zebu, first calving age, calving interval, pure cattle breeds.

\section{Introducción}

El uso de la inseminación artificial a nivel mundial y nacional, ha sido tema de discusión, el grueso de la población de vacas sigue siendo servido a través de monta natural (MN), sin embargo,el uso de la inseminación artificial (IA) está en aumento. Es común conocer opiniones de ganaderos a favor y en contra de la IA, los que están a favor destacan el mejoramiento genético que se favorece a través de la IA, los que están en contra, dicen que se obtienen mejores rendimientos de preñez a través de la MN y que demanda menos trabajo, esto último sobre preñez lo confirma Lima et al. (2009).

El anestro es un factor responsable de la baja eficiencia reproductiva de los bovinos en regiones de clima tropical, donde los animales Bos indicus componen la mayor parte de la población (Baruselli et al., 2004). La disminución de la productividad y la precocidad en las razas Bos indicus es consecuencia de una mayor tolerancia a las presiones ambientales (Nogueira, 2004).

Existen parámetros zootécnicos para medir la eficiencia en la reproducción de los animales, por ejemplo, la edad a primer parto (EPP) que consiste en el tiempo que tarda un animal en alcanzar su pubertad, madurez sexual y reproducirse por primera vez (Hare et al., 2006). En el caso de las novillas Brahman, datos de referencia indican que deben alcanzar la pubertad a una edad de dieciocho meses y entrar a servicio a los dos años, con un peso de 320 a 340 kg (Bastidas-Mendoza, 1999). Sin embargo, se considera que en Bos indicus la llegada a la pubertad es tardía tanto en hembras como en machos, atribuido a causas genéticas y de origen ambiental, donde las hembras podrían alcanzar una edad a primer parto hasta los cuarenta meses de edad (Nogueira, 2004).

El intervalo entre partos (IEP) es otro de los parámetros, se refiere al tiempo que transcurre entre un parto y su consecutivo (Instituto Nacional de Innovación y Transferencia en Tecnología Agropecuaria [INTA], 2017). Constituye el carácter más importante en la evaluación de la eficiencia reproductiva de las vacas en el hato, se considera ideal un IEP de 365 días, por lo que una vaca debería producir un ternero por año (Ossa et al., 2007).

Tanto la EPP como el IEP son dos indicadores del comportamiento y manejo reproductivo del hato, ambos son afectados por fuentes de variación genética y no genética (Ossa et al., 2007). La característica de edad al primer parto presenta heredabilidad moderada y correlación positiva con el primer intervalo entre partos (Mercadante et al., 2000), por lo que es necesario prestar atención a la selección por precocidad sexual (Duitama et al., 2013; Pereira et al., 2002). Trabajos confirman una correlación positiva entre EPP e IEP, con valores de 0,55 y 0,32, respectivamente (Estrada-León et al., 2008; Ponce, 2008) y, esta correlación indica que vacas que paren por primera vez a menor edad tendrán un mejor desempeño reproductivo subsecuente, o sea, intervalos entre partos más cortos.

Al contrario de la EPP, la heredabilidad del IEP es baja, lo cual indica que el mejoramiento de este carácter depende, básicamente, de mejores condiciones de alimentación, sanidad, manejo reproductivo y decisiones 
administrativas, con el objetivo de que las vacas se recuperen rápidamente del parto y de los primeros meses de lactancia (Galeano y Manrique, 2010; Montes et al., 2009). En todo caso, tanto la EPP como el IEP han demostrado ser indicadores que tienen impacto económico sobre la productividad de las fincas (Martínez, 2009).

En Costa Rica, para la población de ganado Bos indicus con certificado de registro genealógico de la Asociación de Criadores de Ganado Cebú (ASOCEBÚ), no se contaba con estudios recientes dirigidos hacia la evaluación de los principales parámetros de interés zootécnico, por lo que no existe una caracterización reproductiva con datos de edad a primer parto, intervalo entre partos y porcentajes de preñez.

El objetivo de esta investigación fue caracterizar los métodos de reproducción utilizados en las razas puras cebuínas y calcular la EPP e IEP de una población Brahman gris pura en Costa Rica.

\section{Materiales y métodos}

Para caracterizar los métodos de reproducción utilizados en las razas puras cebuínas y calcular la edad a primer parto e intervalo entre parto de una población Brahman gris pura en Costa Rica, se siguió la siguiente metodología:

\section{Métodos de reproducción (natural o variantes artificiales) utilizados para reproducir la población de animales activos inscritos en ASOCEBÚ}

Se utilizó la base de datos de la población de ganado activa (animales presentes en finca y provenientes de ganaderías que inscribieron al menos un animal entre enero de 2010 y diciembre de 2017) con certificado de registro genealógico de la Asociación de Criadores de Ganado Cebú de Costa Rica (ASOCEBÚ); la cual fue actualizada al mes de marzo del año 2018, producto del inventario físico nacional realizado por ASOCEBÚ. Se evaluaron datos de 10995 animales de las razas Brahman Gris, Nelore, Brahman Rojo, Gyr, Guzerat e Indubrasil. De esta base de datos se extrajo la distribución de los métodos de reproducción utilizados en esta población de ganado puro (monta natural, inseminación artificial, transferencia de embriones producidos in vivo e in vitro).

\section{Determinación de parámetros reproductivos en la raza Brahman}

Se calcularon los parámetros reproductivos de la edad a primer parto (EPP) y el intervalo entre partos (IEP), de tres de las ganaderías que más aportaban ganado Brahman Gris al Registro Nacional de la ASOCEBÚ, haciendo uso de los datos almacenados por la Asociación a través del registro de nacimientos. En principio, la idea era contar con los registros de finca para poder calcular también parámetros como edad a primer servicio, servicios por concepción, porcentajes de preñez, pérdidas gestacionales, parición y vida productiva, sin embargo, no se obtuvo acceso a dichos registros, por tanto, el análisis de parámetros reproductivos fue restringido. Las tres ganaderías a las que se les solicitó y que autorizaron el uso de los registros de la Asociación, se dedicaban a la cría de la raza Brahman Gris y se ubicaban en las regiones Huetar Atlántica, Brunca y Chorotega de Costa Rica.

De manera arbitraria, las ganaderías se identificaron como Finca 1, 2 y 3 . La ganadería identificada como Finca 1, aportó 2547 datos desde enero del año 2007 hasta abril del año 2018. Para la estimación del intervalo entre partos (IEP) y edad al primer parto (EPP), se utilizaron 682 registros de nacimientos. La ganadería identificada como Finca 2, aportó 4403 datos desde enero del año 1950 a abril del año 2018 y para el análisis del IEP y EPP se hizo uso de un total de 2813 registros. La ganadería identificada como Finca 3, aportó 5792 nacimientos registrados desde enero del año 1950 a febrero del año 2018, de los cuales se utilizaron 4647. En todos los casos, la disminución entre el aporte y la cantidad de registros usados, se debió a la edición y filtrado de la base de datos, para garantizar confiabilidad de los resultados, asimismo, se descartaron los nacimientos de las vacas que tuvieron su primera cría antes de 1990, debido a la falta de información en cuanto a fechas de nacimiento. 
La base de datos contempló el total de nacimientos registrados en ASOCEBÚ para las tres ganaderías en estudio. Esta base de datos incluyó animales inscritos en cada finca, progenie traspasada o vendida y crías de vacas vendidas en estado de preñez. Las fincas seleccionadas representaron el 24,4 \% de la población Bos indicus activa de la Asociación y el 32,7 \% de la población de la raza Brahman Gris registrada.

Se obtuvieron 8610 registros de nacimientos de la raza Brahman Gris. Se clasificaron los nacimientos de acuerdo con el método utilizado para reproducción (animales nacidos por fertilización in vitro, transferencia de embrión, monta natural o inseminación artificial). Se evaluaron los parámetros de EPP e IEP para nacimientos producto de monta natural e inseminación artificial de manera general e individual (por método de reproducción), la base de datos completa sobre IEP era de 5712 registros, a la hora de separar el IEP por método reproducción, se eliminaron 824 de ellos, esto para filtrar con certeza aquellos IEP que provenía de uno u otro método de reproducción, quedando 2755 registros de IEP de animales reproducidos únicamente por medio de IA y 2133 de MN.

La información se analizó a través de estadística descriptiva (porcentajes, correlación, promedios, desviación estándar e intervalos de confianza al 95 \%) con el uso de tablas dinámicas en Microsoft Office Excel ${ }^{\circledR}$ y del paquete estadístico SPSS v22.

\section{Resultados}

\section{Nacimientos de acuerdo con el método de reproducción (cubrición natural o variantes artificiales)}

La distribución de animales nacidos de acuerdo con el método de reproducción de la población activa de ganado Bos indicus con registro genealógico inscritos en ASOCEBÚ al mes de marzo del año 2018 se detalla en el Cuadro 1. Adicionalmente, se dividieron los datos por método de reproducción por raza (Figura 1).

Cuadro 1. Distribución de la población de ganado con registro geneológico en la Asociación de Criadores de Ganado Cebú de Costa Rica (ASOCEBÚ) por método de reproducción al mes de marzo, 2018. San José, Costa Rica, 2018.

Table 1. Distribution of the population of cattle with a geneological (genealogical) records in the Association of Breeders of Zebu Cattle (ASOCEBU) by reproduction method to March, 2018. San Jose, Costa Rica, 2018.

\begin{tabular}{lcccc}
\hline Tipo de reproducción & Machos & Hembras & Total de animales & $\begin{array}{c}\text { Distribución } \\
\text { porcentual del total }\end{array}$ \\
\hline Monta natural & 1849 & 4501 & 6350 & 57,8 \\
Inseminación artificial & 768 & 2086 & 2854 & 26,0 \\
FIV & 418 & 669 & 1087 & 9,9 \\
TE & 149 & 555 & 704 & 6,4 \\
Total general & $\mathbf{3 1 8 4}$ & $\mathbf{7 8 1 1}$ & $\mathbf{1 0 9 9 5}$ & $\mathbf{1 0 0 , 0}$ \\
\hline
\end{tabular}

FIV: embriones producidos a través de ovocitos de vacas donantes fertilizados por la técnica in vitro y transferidos a receptoras. TE: embriones producidos por la técnica in vivo y transferidos a receptoras / FIV: embryos produced through oocytes from donor cows fertilized by the in vitro technique and transferred to recipients. TE: embryos produced by the in vivo technique and transferred to recipients. 


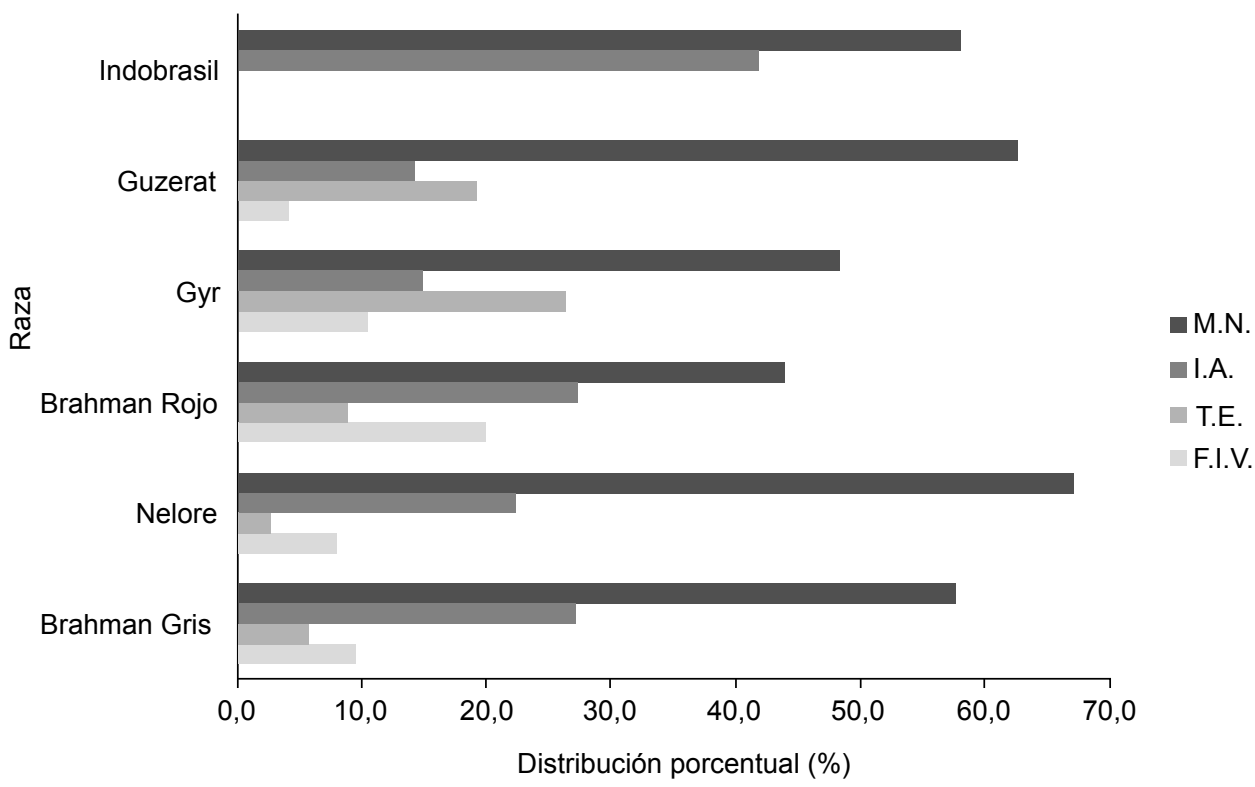

Figura 1. Distribución porcentual de la población activa Bos indicus según raza y método de reproducción. San José, Costa Rica, 2018. M.N.: monta natural, I.A.: inseminación artificial, T.E.: transferencia de embriones, F.I.V.: fertilización in vitro.

Figure 1. Percentage distribution of the Bos indicus active population according to breed and reproduction method. San Jose, Costa Rica. 2018.

M.N.: natural breeding, I.A.: artificial insemination, T.E.: embryo transfer, F.I.V.: in vitro fertilization.

\section{Parámetros reproductivos en la raza Brahman Gris}

\section{Edad a primer parto $(E P P)$}

En promedio, el rango de edad a primer parto (EPP) de las tres fincas fue de 41 a 42,8 meses.

La edad a primer parto (EPP) para cada una de las tres fincas del estudio, así como el promedio general de todos los datos analizados se muestran en el Cuadro 2. La finca más eficiente en este parámetro fue la 2, pero con el menor intervalo de confianza.

Cuadro 2. Valores de edad a primer parto (EPP) obtenidos en hembras de la raza Brahman Gris en tres fincas de ganado con registro genealógico en Costa Rica. San José, Costa Rica, 2018.

Table 2. Age to first calving (EPP) obtained in females of the Gray Brahman breed in three cattle farms with genealogical records in Costa Rica. San Jose, Costa Rica, 2018.

\begin{tabular}{cccccc}
\hline Finca & N & EPP (meses) & D.E. & - IC95 \% & + IC 95 \% \\
\hline 1 & 332 & 41,0 & 12,4 & 39,6 & 42,3 \\
2 & 690 & 38,9 & 9,3 & 38,2 & 39,5 \\
3 & 1384 & 42,8 & 9,2 & 42,3 & 43,3 \\
Promedio & $\mathbf{8 0 2}$ & $\mathbf{4 1 , 4}$ & $\mathbf{9 , 9}$ & $\mathbf{4 1}$ & $\mathbf{4 1 , 8}$ \\
\hline
\end{tabular}

D.E.: desviación estándar; IC: intervalo de confianza / standard deviation; IC: confidence interval. 
La distribución o número de animales dentro de cada rango de EPP fue normal, donde la mayoría de animales mostraron una EEP a edades entre 37,1 y 38 meses (Figura 2). El comportamiento promedio de la EPP a través de los años (1990-2017) para las tres fincas Brahman Gris del estudio mostró su máximo en el año 2017 y el mínimo en 1992 (Figura 3).

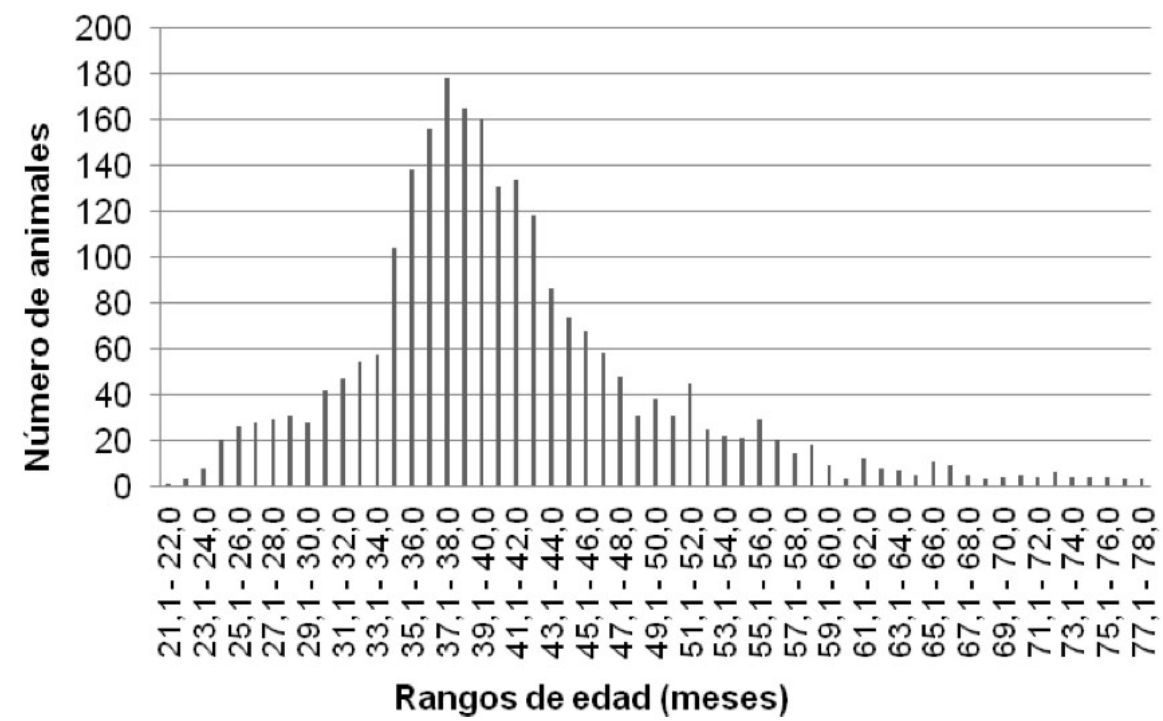

Figura 2. Distribución de la población de tres ganaderías de acuerdo con los rangos de edad a primer parto (EPP), en hembras de la raza Brahman Gris. San José, Costa Rica. 2018.

Figure 2. Population distribution of three livestock farms according to the age ranges for the first calving age (EPP) in females of the Gray Brahman breed. San Jose, Costa Rica. 2018.

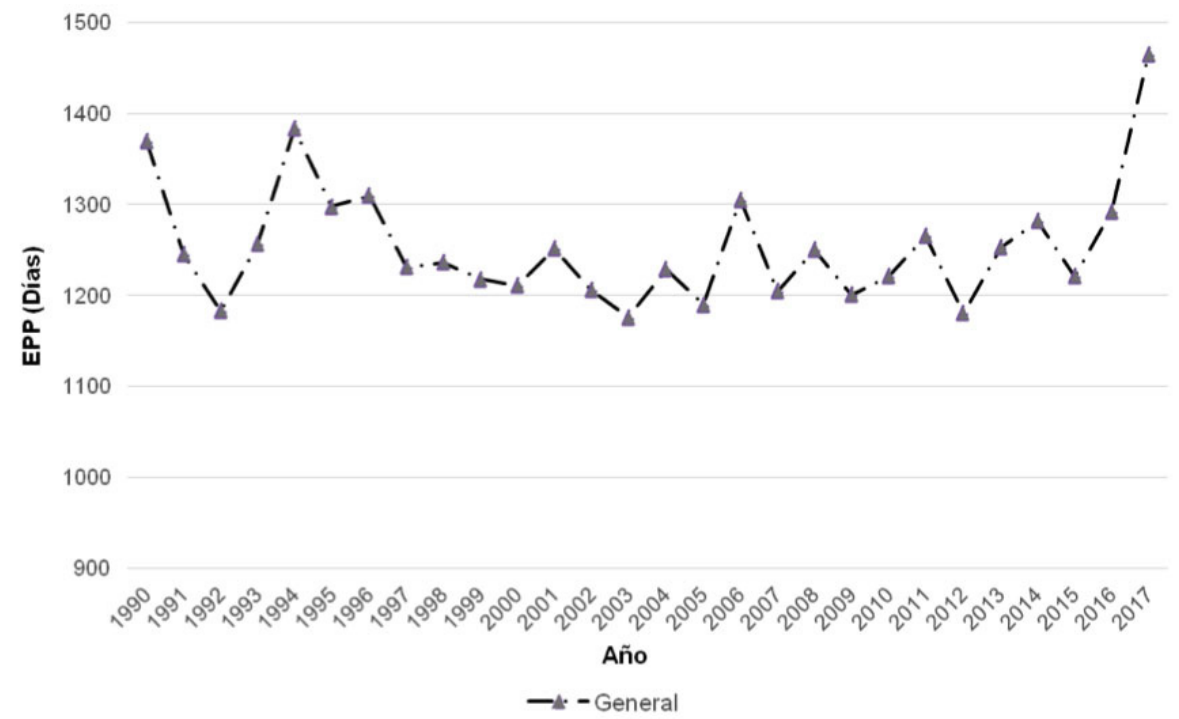

Figura 3. Comportamiento de la variable edad a primer parto (EPP) de acuerdo con el año de nacimiento en hembras de la raza Brahman Gris. San José, Costa Rica, 2018.

Figure 3. Behavior of first calving age (EPP) variable according to the year of birth in females of the Gray Brahman breed. San Jose, Costa Rica, 2018. 
Intervalo entre partos (IEP)

El rango del promedio de intervalo entre parto (IEP) entre las fincas fue de 537,9 a 566,8 días.

El intervalo entre partos (IEP) mostró que las vacas de Finca 3 fueron más eficientes, mientras que la Finca 2 presentó los valores más elevados, independiente del tipo de servicio realizado (Cuadro 3).

Cuadro 3. Valores de intervalo entre parto (IEP) obtenidos en hembras de la raza Brahman Gris en Costa Rica.San José, Costa Rica. 2018.

Table 3. Calving interval (IEP) values obtained in females of the Gray Brahman breed in Costa Rica. San Jose, Costa Rica. 2018.

\begin{tabular}{cccccc}
\hline Finca & N & IEP (días) & D.E. & - IC95\% & + IC 95\% \\
\hline 1 & 349 & 542,8 & 261,1 & 515,3 & 570,3 \\
2 & 2123 & 566,8 & 196,2 & 558,4 & 575,1 \\
3 & 3263 & 537,9 & 167,5 & 532,2 & 543,7 \\
Promedio & $\mathbf{1 9 1 1 , 7}$ & $\mathbf{5 4 8 , 9}$ & $\mathbf{1 8 5 , 8}$ & $\mathbf{5 4 4 , 1}$ & $\mathbf{5 5 3 , 7}$ \\
\hline
\end{tabular}

D.E.: Desviación estándar / standard deviation. IC: intervalo de confianza / confidence interval.

La Figura 4 muestra la distribución de la población de las tres fincas de ganado Brahman Gris en cuanto al número de animales dentro de cada rango de IEP definido. Se identificó que la mayoría de animales mostraron un IEP entre los 13 y 14,1 meses.

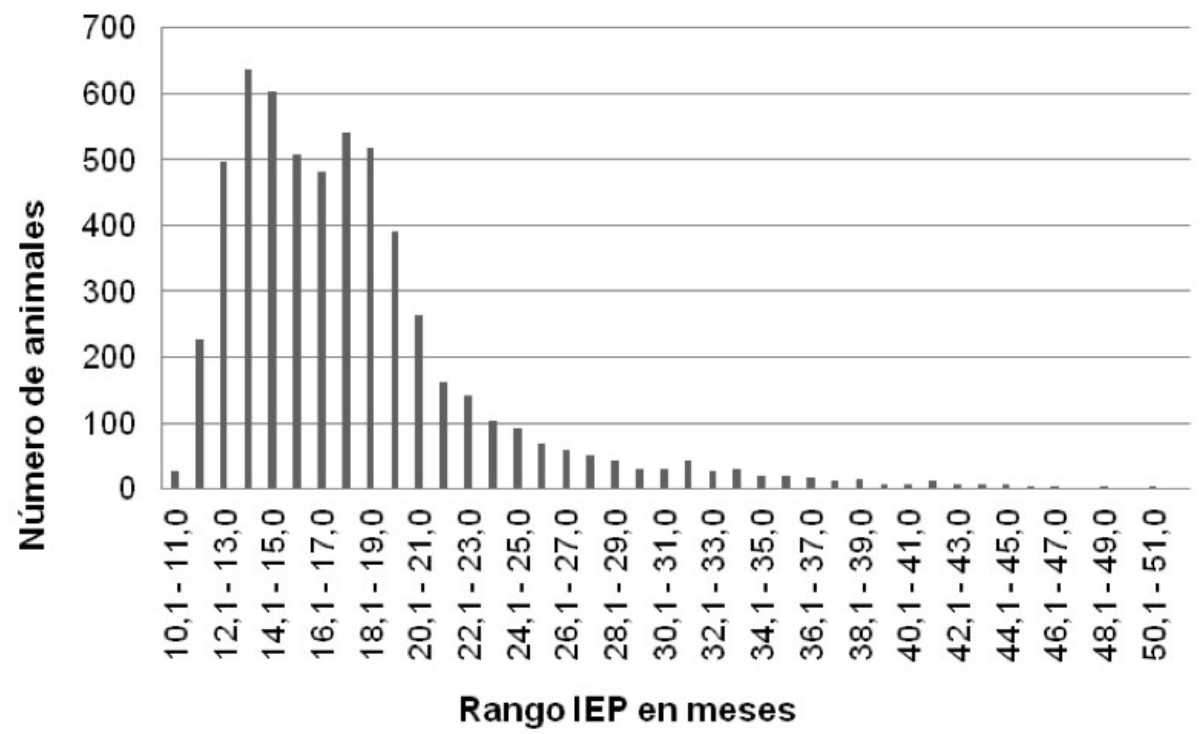

Figura 4. Distribución de la población de tres ganaderías de acuerdo con los rangos de edad para la variable intervalo entre parto (IEP) en hembras de la raza Brahman Gris. San José, Costa Rica. 2018.

Figure 4. Population distribution of three livestock farms according to the age ranges for the calving interval (IEP) variable in females of the Gray Brahman breed. San Jose, Costa Rica. 2018. 
Los valores promedio de intervalo entre parto (IEP) por número de parto por finca para la población de animales de las tres fincas de la raza Brahman Gris del estudio. Conforme aumentó el número de parto disminuyó el número de registros, lo cual evidenció la selección de los animales o el cumplimiento de la vida productiva (Cuadro 4 y Figura 5).

Cuadro 4. Valores de intervalo entre parto (IEP) por número de parto de las tres fincas en hembras de la raza Brahman Gris. San José, Costa Rica. 2018.

Table 4. Calving interval (IEP) values by calving number of the three farms in females of the Gray Brahman breed. San Jose, Costa Rica. 2018.

\begin{tabular}{|c|c|c|c|c|c|c|}
\hline Finca & Parto & $\mathbf{N}$ & Media & D.E. & - IC $95 \%$ & + IC $95 \%$ \\
\hline \multirow[t]{6}{*}{1} & $1-2$ & 160 & 572,8 & 276,5 & 529,6 & 616,0 \\
\hline & $2-3$ & 85 & 506,1 & 215,6 & 459,6 & 552,6 \\
\hline & $3-4$ & 53 & 568,9 & 295,8 & 487,3 & 650,4 \\
\hline & $4-5$ & 28 & 461,5 & 194,7 & 386,0 & 537,0 \\
\hline & $5-6$ & 14 & 403,1 & 95,1 & 348,2 & 458,1 \\
\hline & $6-7$ & 6 & 700,5 & 376,5 & 305,3 & 1095,7 \\
\hline \multirow[t]{10}{*}{2} & $1-2$ & 515 & 624,0 & 224,6 & 604,6 & 643,4 \\
\hline & $2-3$ & 413 & 565,4 & 180,7 & 547,9 & 582,9 \\
\hline & $3-4$ & 356 & 546,0 & 182,9 & 526,9 & 565,1 \\
\hline & $4-5$ & 284 & 555,2 & 212,3 & 530,4 & 580,0 \\
\hline & $5-6$ & 217 & 539,0 & 183,6 & 514,4 & 563,5 \\
\hline & $6-7$ & 143 & 549,3 & 185,1 & 518,7 & 579,9 \\
\hline & $7-8$ & 96 & 505,0 & 100,6 & 484,6 & 525,4 \\
\hline & $8-9$ & 51 & 539,4 & 172,8 & 490,8 & 588,0 \\
\hline & $9-10$ & 28 & 501,2 & 88,2 & 467,0 & 535,4 \\
\hline & $10-11$ & 14 & 562,1 & 107,1 & 500,3 & 624,0 \\
\hline \multirow[t]{9}{*}{3} & $1-2$ & 995 & 578,2 & 177,0 & 567,2 & 589,2 \\
\hline & $2-3$ & 769 & 541,3 & 183,9 & 528,3 & 554,3 \\
\hline & $3-4$ & 572 & 522,3 & 159,4 & 509,2 & 535,3 \\
\hline & $4-5$ & 419 & 517,2 & 141,3 & 503,6 & 530,8 \\
\hline & $5-6$ & 276 & 493,1 & 119,9 & 478,8 & 507,3 \\
\hline & $6-7$ & 143 & 492,1 & 134,3 & 469,9 & 514,3 \\
\hline & $7-8$ & 52 & 471,7 & 115,4 & 439,6 & 503,8 \\
\hline & $8-9$ & 28 & 484,5 & 197,1 & 408,0 & 560,9 \\
\hline & $9-10$ & 9 & 432,7 & 108,5 & 349,2 & 516,1 \\
\hline
\end{tabular}

D.E.: desviación estándar; IC: intervalo de confianza / D.E.: standard deviation; IC: confidence interval. 


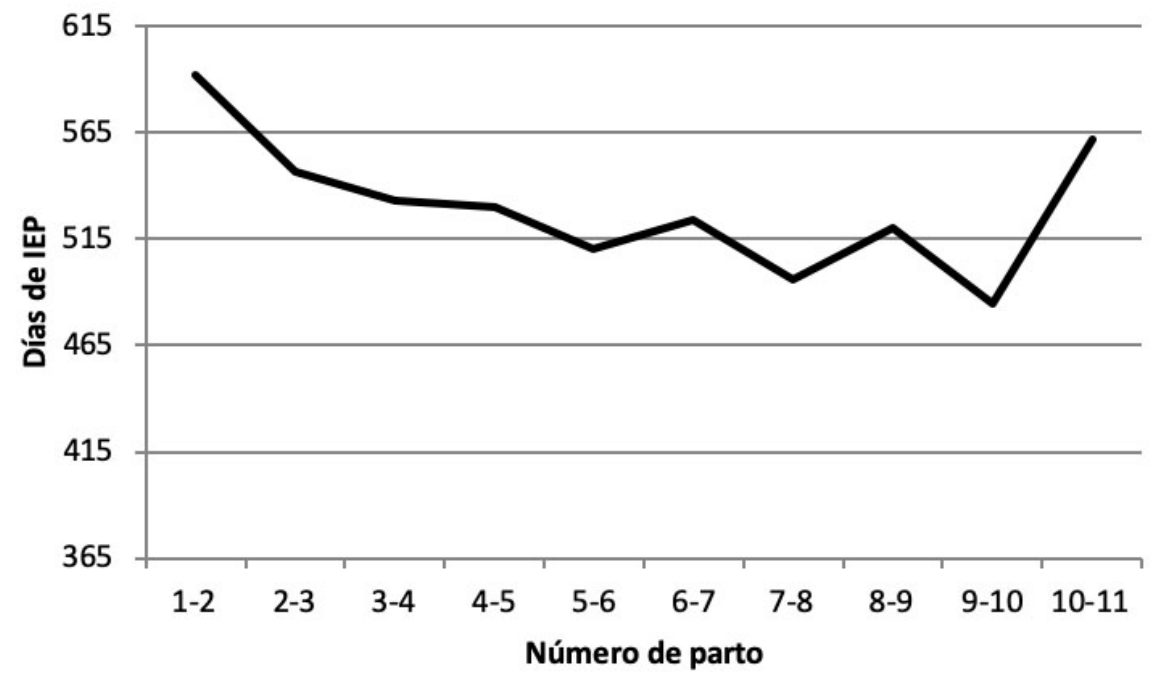

Figura 5. Comportamiento promedio del intervalo entre parto (IEP) de vacas de raza Brahman gris de tres fincas con registro genealógico. San José, Costa Rica. 2018.

Figure 5. Average calving interval (IEP) behavior between calving the Grey Brahman breed cattle in three farms with genealogical records. San Jose, Costa Rica. 2018.

Los datos de IEP de las tres fincas utilizadas para el análisis de la raza Brahman Gris, se clasificaron por tipo de cubrición o servicio, es decir, se clasificaron en IEP originados a partir de servicios exitosos consecutivos por medio de monta natural o inseminación artificial (Figura 6). Por monta natural el IEP fue mayor en comparación con el uso de inseminación artificial.

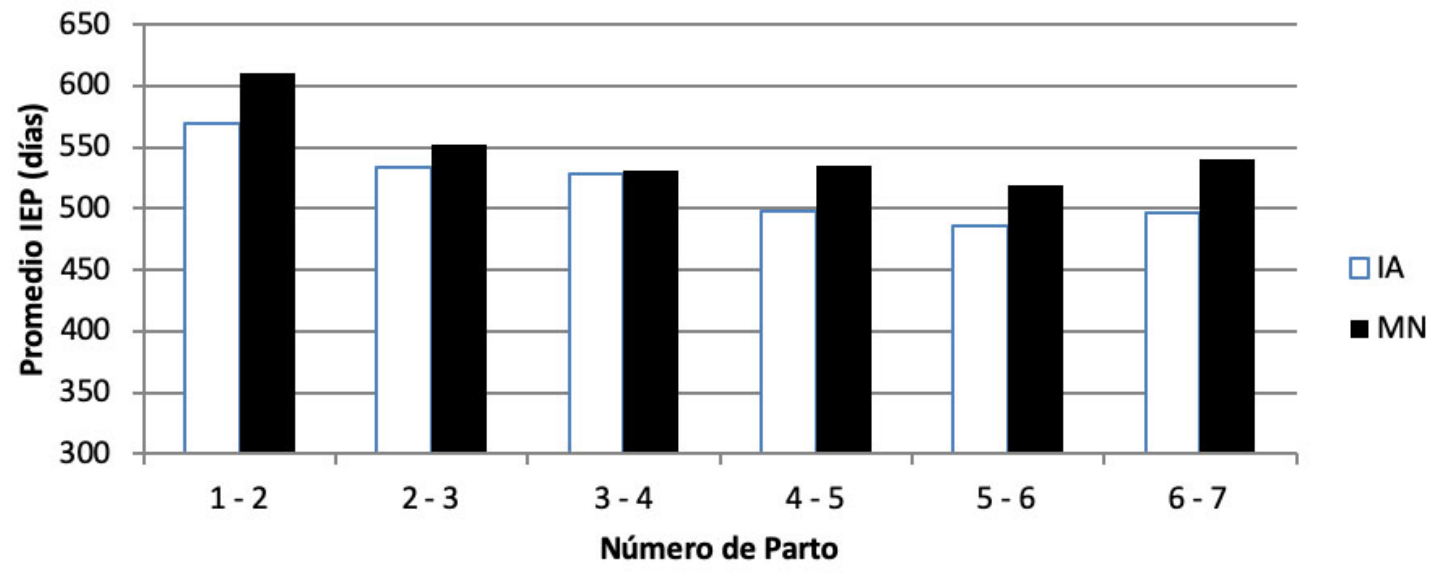

Figura 6. Promedio del intervalo entre parto (IEP) de vacas de raza Brahman gris de tres fincas con registro genealógico, clasificados por tipo de servicio (IA: inseminación artificial o MN: monta natural). San José, Costa Rica. 2018.

Figure 6. Calving interval (IEP) average of the Grey Brahman breed cattle in three farms with genealogical records, classified by type of service (AI: artificial insemination or MN: natural breeding). San Jose, Costa Rica. 2018. 


\section{Discusión}

En cuanto a los nacimientos por método de reproducción, el más utilizado en la población evaluada fue la monta natural (MN) (57,8\%), seguida por la técnica de inseminación artificial (IA) (26\%), lo cual es congruente con los datos generados por la Encuesta Ganadera 2004 (Corporación Ganadera [CORFOGA], 2005), y contrario a lo reportado en ganado lechero de Estados Unidos, donde el 26,8 \% eran servicios por monta natural y 72,5 \% por inseminación articial (United States Department of Agriculture [USDA], 2009).

En este análisis se observó que la producción y transferencia de embriones, sea in vitro o in vivo, era poco utilizada, a pesar de ello, su uso fue mayor en comparación con las lecherías comerciales de Estados Unidos donde apenas fue de $0,7 \%$ (United States Department of Agriculture [USDA], 2009), quizá esta diferencia se debe a las formas de manejo entre ganado puro y comercial. La alta inversión en la utilización de biotecnologías reproductivas, debida a los costos por tratamientos de superovulación, sincronización de donadoras, aspiración folicular, preparación de receptoras, la inseminación, la colecta de embriones o cultivo de ovocitos y embriones y su transferencia, hacen que dichas tecnologías sean menos utilizadas en la cría comercial (Bolívar y Maldonado, 2008). Generalmente, las técnicas que implican mayor inversión económica como la FIV y TE (Bolívar y Maldonado, 2008), son más utilizadas en razas cuya demanda va en aumento y que presentan una baja población (Romo, 1993; Salgado et al., 2011), tal es el caso de las razas Gyr, Guzerat y Brahman Rojo, donde se evidenció un porcentaje importante del uso de estas técnicas.

El intervalo entre partos (IEP) promedio de esta investigación (41-42,8 meses) es el reporte más recurrente, ya que en el país no se cuenta con estudios recientes sobre este parámetro en hembras Bos indicus con registro genealógico, en este sentido, en 2009, Martínez (2009) reportaron un intervalo entre 30 y 36 meses de EPP en ganado de carne. En hembras cruzadas (Bos taurus $x$ Bos indicus) con predominio de la raza Brahman, WingChingJones (2017) indicó promedios de EPP de 31,2 meses en la zona sur del país. Se ha informado de resultados de EPP de 29,3 meses en la raza Jersey (Castillo-Badilla et al., 2013) y de 29,8 a 30,7 meses para la raza Holstein (Cascante, 2008; Salazar-Carranza et al., 2013). Hace 45 años, Bazán (1974) publicó valores promedio de 42 meses para hembras Brahman en la zona Pacífica de Costa Rica. En otros países, menores valores fueron mostrados por Mora (2005) y Duitama et al. (2013) quienes realizaron estudios en Colombia, y encontraron datos de 37 7,2 meses

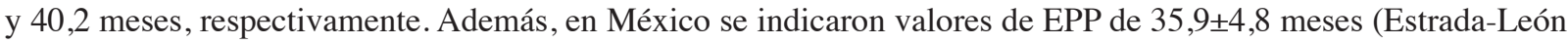
et al., 2008). En Colombia, para una población Brahman de juzgamiento se reportaron valores de EPP de 33,4 \pm 0,72 meses (Carrizales, 2005).

Si se toma como referencia los valores de EPP reportados en la literatura nacional y los resultados de este estudio (Figura 3), el progreso genético y productivo observado es mínimo. Diversos factores afectan la EPP en las razas Bos indicus, entre los que destacan la genética, la nutrición, el manejo y el ambiente (Baca et al., 1998; Nogueira, 2004; Pereira, 2000). Desde el punto de vista genético, se ha encontrado que las razas europeas (Bos taurus) son más precoces que las razas cebuínas (Bos indicus) (Silva et al., 2002), de ahí que la madurez sexual en las novillas Brahman es más tardía que en las novillas Bos taurus (Carrizales, 2005; Magaña y Segura-Correa, 2001). Sin embargo, los datos encontrados mostraron que un $8,8 \%$ de la población presentó su edad a primer parto antes de los treinta meses de edad, lo cual es un indicativo de que existe potencial para el mejoramiento de este parámetro tanto por vía genética como de manejo.

Las prácticas de alimentación inadecuadas y la baja calidad nutricional de los forrajes en el trópico afectan la EPP. Una adecuada nutrición post destete permite lograr una disminución en la edad de la pubertad y en la EPP (Patterson et al., 1992), debido a que la ingesta de nutrientes de acuerdo con los requerimientos nutricionales resulta en un mayor peso al momento de la pubertad (Oyedipe et al., 1982). Además, las novillas más pesadas al destete y que mantienen niveles adecuados de nutrición post destete, alcanzan la pubertad a una edad más temprana (Barcellos et al., 2014; Endecott et al., 2013; Greer et al., 1983). 
La ingesta dietética reducida da como resultado la incapacidad de ovular, debido a los niveles insuficientes de hormona LH circulante para estimular la maduración del folículo dominante (Nogueira, 2004). Otros aspectos de manejo también inciden la EPP, la selección genética de hembras precoces (Nogueira, 2004), el uso de sementales seleccionados por circunferencia escrotal (Velázquez et al., 2004) y la estimulación hormonal de las novillas son prácticas que podrían disminuir la EPP (Bastidas-Mendoza, 1999). Por el contrario, poco o nulo control sobre los animales durante la lactancia y la etapa entre el destete y la pubertad, puede repercutir en un bajo desempeño reproductivo (Le Neindre et al., 1996). Las estrategias de manejo deberían enfocarse hacia la fase post destete-apareamiento, lo cual implica la adecuación del genotipo y el ambiente, a fin de asegurar un desempeño reproductivo aceptable (Bastidas-Mendoza, 1999).

Los valores para el intervalo entre partos (IEP) ( $\pm 537,9$ a 566,8) son mayores a datos reportados en

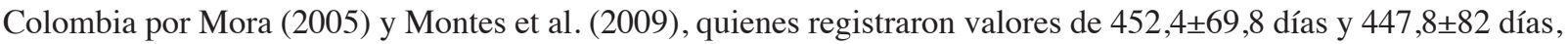
respectivamente; mientras que en México se encontraron valores de 456 125 días (Estrada-León et al., 2008). En Costa Rica, el intervalo parto-concepción (días abiertos) que reportó Martínez (2009) fue de 155 días, lo que aproximadamente generaría un IEP de 438 días.

En Guanacaste, Costa Rica, datos publicados por Castillo (2008), indicaron un IEP de 482,8 días en la raza Brahman y 450,5 días en animales media sangre Brahman. Las evaluaciones de IEP en la raza Brahman corresponden a siete trabajos de graduación realizados en la Escuela de Zootecnia de la Universidad de Costa Rica, entre los años 1979 a 1994 (WingChing-Jones, 2017). Estudios reportaron valores de IEP de 15 meses (456,5 días) en la zona Atlántica y 16 meses (487 días) en la zona Pacífica en hembras Brahman (Bazán, 1974); además, se encontró un IEP de 454,6 $( \pm 91,9)$ días en hembras Bos taurus X Bos indicus, en la zona sur de Costa Rica (WingChing-Jones, 2017).

En general, en este estudio, se encontró una amplia dispersión de los valores de IEP, con un valor mínimo y máximo de 10,1 y 51 meses, respectivamente. Un rango amplio de IEP fue también encontrado por WingChingJones (2017), con valores que fueron desde 0,8 hasta los 3 años en bovinos de carne en la zona sur de Costa Rica. Factores ambientales, nutricionales, endocrinos, genéticos y de manejo han sido relacionados con la variación en el IEP (Carrizales, 2005). En el caso de las fincas 1 y 2, se conoció que utilizan biotecnologías reproductivas (FIV, TE) que requieren de manejos específicos que en ocasiones tienden a aumentar los IEP, aunque conlleva a beneficios como mejora genética en plazos menores y sincronización en las mejores épocas de preñez o parición.

Para todas las fincas, el intervalo entre el primer y segundo parto fue mayor, luego el IEP disminuyó conforme aumentó el número de partos hasta que entre el 6 y 7 parto se dio un aumento, quizá el cambio en el comportamiento, además del desgaste del animal, se debe a un menor número de eventos registrados.

El comportamiento del IEP que se presenta en la Figura 5, es similar a lo reportado en Colombia por Carrizales (2005), donde, en hembras de la raza Brahman participantes de juzgamiento, el IEP promedio entre el primer y

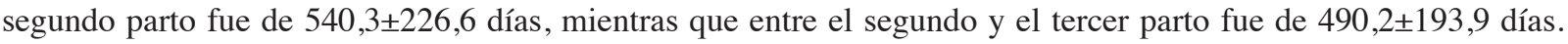
Este autor indicó que esta reducción en la duración del intervalo se mantuvo hasta el quinto parto y se estabilizó en valores similares hasta el octavo parto; a partir de ahí, mostró un incremento. También en hembras del cruce Bos taurus $\mathrm{X}$ Bos indicus se ha observado que el intervalo del primero al segundo parto es mayor, esto porque de primer y segundo parto todavía no tienen el desarrollo corporal completo, lo cual disminuye su eficiencia reproductiva, además, la demanda de leche por parte de sus crías las somete a un estrés ambiental (Arellano et al., 2006; Hernández-Reyes et al., 2001).

El comportamiento promedio del IEP para las vacas Brahman Gris, independientemente del tipo de cubrición utilizado, confirmó que los animales primerizos fueron la categoría animal más difícil de preñar. Esto coincide con los resultados en ganado cebuíno de Segura-Correa et al. (2017) donde la misma categoría de vacas obtuvo el menor porcentaje de concepción, comparado con aquellos de dos o más partos, lo que siginifica un aumento en los días abiertos e IEP. 
Los resultados mostrados en la Figura 6 reflejaron el comportamiento del intervalo entre parto (IEP) de las vacas servidas únicamente a través de $\mathrm{MN}$ o de IA, este tipo de análisis donde se compara el manejo reproductivo no es común, se ha encontrado información del desempeño productivo y reproductivo de animales originados por medio de IA o MN, los cuales han sido similares (Ocaña-Martínez y Alvarado-Cerón, 2017), pero no sobre el parámetro categorizado por tipo de cubrición. En este estudio fue evidente que el IEP a través de los diferentes partos fue favorable para el grupo reproducido por medio de IA, lo cual brinda un impulso hacia el uso de este tipo de reproducción. Se ha demostrado que la IA es una opción que genera mejores créditos económicos que la MN y que la evaluación debe ser integral, no únicamente relegada al porcentaje de preñez (Dos-Santos et al., 2018).

\section{Conclusiones}

La monta natural sigue siendo el método de reproducción más utilizado en la población de ganado con registro genealógico de Costa Rica. Esto aplicó para la población general de cebú, así como para cada una de las razas de la Asociación de Criadores de Ganado Cebú de Costa Rica (ASOCEBÚ).

De las tecnologías de reproducción asistida que se aplican a nivel comercial, la inseminación artificial fue la más utilizada. El uso de FIV o TE difiere entre las razas de la ASOCEBÚ.

El resultado de la evaluación de EPP fue de edades altas, lo cual sugiere la necesidad de seleccionar hembras de reemplazo precoces y darles el ambiente adecuado para su desarrollo.

Los valores generales de IEP mostraron un espacio de mejora amplio para optimizar la producción de estos animales.

Los resultados en términos de IEP a favor de la IA pueden deberse a garantía de calidad seminal, implementación de controles que no se dan cuando se usa MN, al efecto de protocolos para sincronización artificial u otros factores, pero es evidente que la inseminación artificial es una herramienta de manejo reproductivo que favorece la disminución del IEP.

Los datos de EPP e IEP de este estudio difirieron de los ideales utilizados en proyecciones de hato.

Durante el desarrollo de este estudio se evidenció carencia de investigaciones en temas de reproducción de los animales Bos indicus con certificado de registro genealógico en Costa Rica. Se requiere generar información sobre tendencias genéticas, parámetros productivos y reproductivos que sirvan como base para la mejora de los hatos.

\section{Agradecimientos}

Los autores agradecen a la Asociación de Criadores de Ganado Cebú de Costa Rica por brindar las facilidades para el desarrollo de este proyecto.

\section{Referencias}

Arellano, S., Martínez, J., Romero, E., Briones, F., Domínguez, M., \& De la Garza, F. (2006). Factores genético-ambientales que afectan el intervalo entre partos y días a primer parto en ganado de doble propósito en el norte de Veracruz. Avances en Investigación Agropecuaria, 10(1), 43-53.

Baca, J. R., Pérez, E., \& Galina, C. S. (1998). Reproductive performance of Bos taurus $\times$ Bos indicus heifers following artificial insemination after spontaneous estrus in the dry tropics of Costa Rica. Veterinaria México, 29(1), 57-66. 
Barcellos, J., Pereira, G., Dias, E., Mcmanus, C., Canellas, L., Bernardi, M., Tarouco, A., \& Prates, E. (2014). Higher feeding diets effects on age and liveweight gain at puberty in crossbred Nelore $\times$ Hereford heifers. Tropical Animal Health and Production, 46(6), 953-960. https://doi.org/10.1007/s11250-014-0593-6

Baruselli, P. S., Reis, E. L., Marques, M. O., Nasser, L. F., \& Bó, G. A. (2004). The use of hormonal treatments to improve reproductive performance of anestrous beef cattle in tropical climates. Animal Reproduction Science, 82-83, 479-486. https://doi.org/10.1016/j.anireprosci.2004.04.025

Bastidas-Mendoza, P. S. (1999). Pubertad en novillas y toros Brahman. Revista de la Facultada de Agronomía (LUZ), 16, $690-707$.

Bazán, O. A. (1974). Diagnóstico del comportamiento reproductivo del ganado de carne en fincas de Costa Rica [Tesis Magister Scientiae, Instituto Interamericano de Ciencias Agrícolas de la OEA]. Repositorio CONARE. http://kimuk.conare. ac.cr/Record/RCATIE_cbcb3d7997a90d4046ba8f47ffda171f

Bolívar, P. A., \& Maldonado, J. G. E. (2008). Análisis de costos de esquemas de transferencia de embriones bovinos utilizados en Colombia. Revista Colombiana de Ciencias Pecuarias, 21(3), 351-364.

Carrizales, J. J. M. (2005). Relación entre la edad, el peso y la eficiencia reproductiva en hembras Brahman de competencia [Tesis de Licenciatura no publicada]. Universidad de La Salle.

Cascante, A. M. A. (2008). Efecto de la edad a primer parto sobre algunas variables productivas, primer intervalo entre partos y características de la curva de lactancia de vacas lecheras en la zona norte de Costa Rica [Tesis de Licenciatura, Universidad de Costa Rica]. Repositorio UCR. http://repositorio.sibdi.ucr.ac.cr:8080/jspui/handle/123456789/2245

Castillo, M. Á. (2008). Propuesta para el manejo reproductivo y genético de la finca de ganado bovino de cría "El Laberinto" en el cantón de Cañas, Guanacaste [Tesis de Licenciatura, no publicada]. Universidad de Costa Rica.

Castillo-Badilla, G., Salazar-Carranza, M., Murillo-Herrera, J., \& Romero-Zúñiga, J. J. (2013). Efecto de la edad al primer parto sobre parámetros productivos en vacas Jersey de Costa Rica. Agronomía Mesoamericana, 24(1), 177-187. https://doi. org/10.15517/am.v24i1.9796

Corporación Ganadera [CORFOGA]. (2005). Encuesta ganadera 2004. http://www.mag.go.cr/biblioteca_virtual_animal/ Encuesta-Ganadera-2004.pdf

Dos-Santos, G., R. Tortorella, \& D. Fausto. (2018). Rentabilidade da monta natural e inseminação artificial em tempo fixo na pecuária de corte. Revista IPecege, 4(1), 28-32. https://doi.org/10.22167/r.ipecege.2018.1.28

Duitama, O., González H. L., Garcia, D., Farah, M., \& Da Fonseca, R. (2013). Productividad acumulada y su relación genética con características reproductivas en hembras Brahman. Revista MVZ Córdoba, 18, 3658-3664. https://doi. org/10.21897/rmvz.132

Endecott, R. L., Funston, R. N., Mulliniks, J. T., \& Roberts, J. (2013). JOINT ALPHARMA-BEEF SPECIES SYMPOSIUM: Implications of beef heifer development systems and lifetime productivity. Journal of Animal Science, 91(3), 13291335. https://doi.org/10.2527/jas.2012-5704

Estrada-León, R. J. R. J., Segura-Correa., J. C., \& Magaña, J. G. (2008). Parámetros Genéticos para caracteres reproductivos de vacas Brahman en un hato del sureste de México. Tropical and Subtropical Agroecosystems, 8(3), $259-263$.

Galeano, A. P., \& Manrique, C. (2010). Estimación de parámetros genéticos para características productivas y reproductivas en los sistemas doble propósito del trópico bajo Colombiano. Revista de la Facultad de Medicina Veterinaria y de Zootecnia, 57(2), 119-131. 
Greer, R. C., Whitman, R. W., Staigmiller, R. B., \& Anderson, D. C. (1983). Estimating the impact of management decisions on the occurrence of puberty in beef heifers. Journal of Animal Science, 56(1), 30-39. https://doi.org/10.2527/ jas1983.56130x

Hare, E., Norman, H. D., \& Wright, J. R. (2006). Trends in calving ages and calving intervals for dairy cattle breeds in the United States. Journal of Dairy Science, 89(1), 365-370. https://doi.org/10.3168/jds.S0022-0302(06)72102-6

Hernández-Reyes, E., Segura-Correa, V. M., Segura-Correa, J. C., \& Osorio-Arce, M. M. (2001). Intervalo entre partos, duración de la lactancia y producción de leche en un hato doble propósito en Yucatán, México. Agrociencia, 35, 699-705.

Instituto Nacional de Innovación y Transferencia en Tecnología Agropecuaria [INTA. (2017). Manual de manejo: Sistemas intensivos sostenibles de ganadería de leche. http://www.mag.go.cr/bibliotecavirtual/L01-10927.pdf

Le Neindre, P., Boivin, X., \& Boissy, A. (1996). Handling of extensively kept animals. Applied Animal Behaviour Science, 49(1), 73-81. https://doi.org/10.1016/0168-1591(95)00669-9

Lima, F. S., Risco, C. A., Thatcher, M. J., Benzaquen, M. E., Archbald, L. F., Santos, J. E. P., \& Thatcher, W. W. (2009). Comparison of reproductive performance in lactating dairy cows bred by natural service or timed artificial insemination. Journal of Dairy Science, 92(11), 5456-5466. https://doi.org/10.3168/jds.2009-2197

Magaña, J. G., \& Segura-Correa, J. C. (2001). Estimates of breed and heterosis effects for some reproductive traits of Brown Swiss and Zebu-related breeds in South-eastern Mexico. Livestock Research for Rural Development, $13(5), 135$. http://www.lrrd.org/lrrd13/5/maga135.htm

Martínez, J. A. (2009). Análisis del mejoramiento y competitividad en la ganadería de carne [Tesis de Licenciatura, no publicada]. Universidad de Costa Rica.

Mercadante, M. E. Z., Lôbo, R. B., \& Oliveira, H. N. de. (2000). Estimativas de (Co)variâncias entre características de reprodução e de crescimento em fêmeas de um rebanho Nelore. Revista Brasileira de Zootecnia, 29(4), 997-1004. https://doi.org/10.1590/S1516-35982000000400008

Montes, V. D., Vergara, G. O., \& Prieto, M. E. (2009). A note on the estimation of the heritability of calving interval in Brahman cattle. Livestock Research for Rural Development, 21(1), 2. http://www.lrrd.org/lrrd21/1/mont21002.htm

Mora, C. O. H. (2005). Evaluación de la edad al primer parto y su incidencia en la vida productiva y reproductiva de las novillas Brahman [Tesis de Licenciatura, no publicada]. Universidad de La Salle.

Nogueira, G. P. (2004). Puberty in South American Bos indicus (Zebu) cattle. Animal Reproduction Science, 82-83, 361-372. https://doi.org/10.1016/j.anireprosci.2004.04.007

Ocaña-Martínez, H. E. O., \& Alvarado-Cerón, J. S. (2017). Evaluación de los parámetros productivos y reproductivos en vacas doble propósito obtenidas por inseminación artificial y monta natural en la finca El Porvenir en Doncello-Caquetá. FAGROPEC, 9(1), 25-31.

Ossa, G. A., Suárez, M. A., \& Pérez, J. E. (2007). Factores ambientales y genéticos que influyen la edad al primer parto y el intervalo entre partos. Revista Ciencia y Tecnología Agropecuaria, 8(2), 74-80. https://doi.org/10.21930/rcta. vol8_num2_art:97

Oyedipe, E. O., Osori, D. I., Akerejola, O., \& Saror, D. (1982). Effect of level of nutrition on onset of puberty and conception rates of zebu heifers. Theriogenology, 18(5), 525-539. https://doi.org/10.1016/0093-691x(82)90185-6 
Patterson, D. J., Perry, R. C., Kiracofe, G. H., Bellows, R. A., Staigmiller, R. B., \& Corah, L. R. (1992). Management considerations in heifer development and puberty. Journal of Animal Science, 70(12), 4018-4035. https://doi. $\operatorname{org} / 10.2527 / 1992.70124018 x$

Pereira, E., Eler, J. P., \& Ferraz, J. B. S. (2002). Análise genética de características reprodutivas na raça Nelore. Pesquisa Agropecuária Brasileira, 37(5), 703-708. https://doi.org/10.1590/S0100-204X2002000500016

Pereira, J. C. C. (2000). Contribuição genética do Zebu na pecuária bovina do Brasil. Informe Agropecuario, Belo Horizonte, 21(205), 30-38.

Ponce, J. M. (2008). Determinación del intervalo entre partos en un hato puro Brahman, en San Marcos de Colón, departamento de Choluteca, Republica de Honduras [Tesis de Licenciatura, no publicada]. Universidad de San Carlos de Guatemala.

Romo, S. G. (1993). Biotecnología reproductiva: Avances en ganado bovino. Veterinaria México, 24(3), $177-184$.

Salazar-Carranza, M., Castillo-Badilla, G., Murillo-Herrera, J., Hueckmann-Voss, F., \& Romero-Zuñiga, J. J. (2013). Edad al primer parto en vacas Holstein de lechería especializada en Costa Rica. Agronomía Mesoamericana, $24(2), 233$. https://doi.org/10.15517/am.v24i2.12522

Salgado, R. O., Mejía, A. A., \& Suárez, P. S. (2011). Eficiencia de la respuesta superovulatoria del ganado Brahman al protocolo P-24. Revista MVZ Córdoba, 16(2), 2521-2527. https://doi.org/10.21897/rmvz.1016

Segura-Correa, J. C., Magaña, J. G., Ake, J. R., \& Segura-Correa, V. M. (2017). Season and parity number influence the conception rate of zebu breed cows in South-eastern Mexico. Livestock Research for Rural Development, 29(11), 29215. http://www.lrrd.org/lrrd29/11/jose29215.html

Silva, C., Aké, R., \& Valle, R. (2002). Edad y crecimiento a la pubertad en toros Suizo Pardo en condiciones tropicales. Revista Cubana de Ciencia Agrícola, 36(3), 205-210.

United States Department of Agriculture [USDA]. (2009). Reproduction Practices on U.S. Dairy Operations, 2007. https:// www.aphis.usda.gov/animal_health/nahms/dairy/downloads/dairy07/Dairy07_is_ReprodPrac_1.pdf

Velázquez, G. M., Gregory, K. E., Bennett, G. L., \& Vleck, L. D. V. (2004). Parámetros genéticos para circunferencia escrotal, edad a la pubertad en vaquillas y tasa de destete en varias razas de bovinos productores de carne. Técnica Pecuaria en México, 42(2), 159-170.

WingChing-Jones, R. (2017). Índices productivos y reproductivos de fincas de cría de ganado bovino de carne en la zona Sur de Costa Rica. UNED Research Journal, 9(2), 247-256. https://doi.org/10.22458/urj.v9i2.1899 